\title{
VARIAÇÃO DE PESO CORPORAL DE PACIENTES EM DIÁLISE PERITONEAL ${ }^{a}$
}

\author{
Kelly Dayane Stochero VELOZO ${ }^{b}$, Sirlem FERRAZ, \\ Michelle Carvalho RAIMUNDO ${ }^{\mathrm{d}}$, Ana Elizabeth Prado Lima FIGUEIREDO ${ }^{\mathrm{e}}$
}

\section{RESUMO}

Este estudo teve como objetivo principal avaliar a variação de peso corporal de pacientes em diálise peritoneal (DP) durante o primeiro ano de tratamento e, como objetivo secundário, identificar pacientes com critério para síndrome metabólica (SM). Estudo observacional, com 35 pacientes, com o mínimo de um ano em DP, de um hospital universitário. Foram coletados dados quanto à situação clínica e demográfica, à variação de peso corporal e aos critérios para SM. Após doze meses do início da diálise, aproximadamente 40\% dos pacientes apresentava sobrepeso e/ou obesidade. A média de variação de peso corporal após um ano foi de 3,7 kg, sendo que a maior variação foi encontrada nos primeiros três meses, com valores de $2,2 \mathrm{~kg}$. A prevalência de SM foi de $30 \%$. Conclui-se que a variação de peso corporal mais importante aconteceu no primeiro ano de tratamento e há uma alta prevalência de SM nos pacientes em DP.

Descritores: Diálise peritoneal. Ganho de peso. Índice de Massa Corporal.

\section{RESUMEN}

Estudio con objetivo principal evaluar la variación en el peso corporal de los pacientes en diálisis peritoneal (DP) durante el primer año de tratamiento y como secundario identificar a pacientes con criterios de síndrome metabólico (SM). Estudio observacional realizado con 35 pacientes de un hospital universitario. Se recogieron datos sobre la situación clínica y demográfica, el cambio de peso corporal y los criterios para SM de la muestra. Después de 12 meses de empezar la DP, aproximadamente el $40 \%$ de los pacientes mostró sobrepeso y obesidad. La variación promedio de peso corporal después de un año fue de 3,7 Kg y la mayor variación fue en los tres primeros meses, con valores de 2,2 Kg. La prevalencia de SM fue del 30\%. Se concluye que el cambio de peso más importante ocurrió en el primer año de tratamiento y existe una alta prevalencia de SM en pacientes en DP.

Descriptores: Diálisis peritoneal. Aumento de peso. Índice de Masa Corporal

Título: Análisis de cambios en el peso de pacientes en diálisis peritoneal.

\section{ABSTRACT}

This study had as its main objective to assess the variation in body weight of patients on peritoneal dialysis (DP) during the first year of treatment and as a secondary goal to identify patients with criteria for metabolic syndrome (SM). Observational study conducted with 35 patients of a university hospital. Data were collected on the clinical and demographic status, change of body weight and criteria for SM from the sample. After 12 months of initiation of dialysis, approximately $40 \%$ of patients showed overweight and/or obesity. The average variation of body weight after one year was $3.7 \mathrm{Kg}$, and the largest variation was found in the first three months, with values of $2.2 \mathrm{Kg}$. The prevalence of $\mathrm{SM}$ was $30 \%$. It was concluded that the most important change of body weight happened in the first year of treatment and there is a high prevalence of SM in patients on DP.

Descriptors: Peritoneal dialysis. Weight gain. Body Mass Index

Title: Assessment of body weight changes in patients on peritoneal dialysis.

\footnotetext{
a Artigo originado do Trabalho de Conclusão do Curso de Graduação em Enfermagem apresentado em 2010 na Faculdade de Enfermagem, Nutrição e Fisioterapia da Pontifícia Universidade Católica do Rio Grande do Sul (PUCRS).

b Enfermeira, Mestranda em Pediatria/Saúde da Criança da PUCRS, Porto Alegre, Rio Grande do Sul, Brasil.

c Enfermeira da Unidade de Diálise do Hospital São Lucas da PUCRS, Porto Alegre, Rio Grande do Sul, Brasil.

d Enfermeira do Setor de Organização de Procura de Órgãos do Hospital São Lucas da PUCRS, Porto Alegre, Rio Grande do Sul, Brasil.

e Doutora em Ciências da Saúde, Professora Adjunta da Faculdade de Enfermagem, Nutrição e Fisioterapia da PUCRS, Porto Alegre, Rio Grande do Sul, Brasil.
} 


\section{INTRODUÇÃO}

Aproximadamente $10 \%$ da população brasileira em diálise utiliza diálise peritoneal (DP) como forma de tratamento de substituição renal. Sabe-se que os pacientes em DP ganham peso corporal no entanto, não existem dados consistentes sobre esta temática. Acredita-se que esse ganho de peso corporal seja similar ao que acontece com a população em geral. $\mathrm{O}$ excesso de peso corporal na população brasileira varia conforme a idade, de $8,8 \%$ a $50,8 \%$, sendo que, com o aumento da idade, a taxa de sobrepeso também aumenta ${ }^{(1)}$.

Cerca de 60 a $80 \%$ da glicose proveniente da solução de diálise é absorvida ${ }^{(2)}$, o que acarreta em aumento da glicose circulante, provocando aumento da secreção de insulina pelo pâncreas, caracterizando a hiperinsulinemia. Supõe-se que a sobrecarga calórica, proveniente do dialisato, tem o potencial de agravar as complicações metabólicas e contribuir para o ganho de peso corporal ${ }^{(2)}$.

A insulina estimula a síntese e o armazenamento dos triglicerídeos no tecido adiposo, principalmente em região abdominal, facilitando o ganho de peso corporal ${ }^{(3)}$. O aumento da gordura abdominal leva ao excesso de ácidos graxos livres (AGL), o que contribui para a resistência à insulina. No fígado, os AGL aumentam a produção de glicose, triglicerídeos e lipoproteínas de baixa densidade (VLDL), associada a uma redução de lipoproteínas de alta densidade (HDL) e aumento de lipoproteínas de baixa densidade (LDL) $)^{(4-5)}$.

O transporte de pequenos solutos (uréia, creatinina e glicose) através da membrana peritoneal acontece de forma individualizada. O Teste de Equilíbrio Peritoneal (PET) é utilizado na prática clínica para a identificação do tipo de transporte de cada paciente. Com essa classificação (alto, médio alto, médio baixo e baixo transportador) é possível ajustar a melhor terapia de diálise peritoneal para o paciente ${ }^{(6-7)}$. Os pacientes alto transportadores apresentam uma alta taxa de remoção de solutos, porém uma baixa ultrafiltração por absorver rapidamente a glicose do dialisato. Já os pacientes baixo transportadores apresentam baixa depuração de solutos, mas uma maior capacidade de ultrafiltração, pois a glicose dissipa-se mais lentamente ${ }^{(6)}$. Essas características da membrana peritoneal podem estar envolvidas no ganho de peso corporal durante o tratamento de DP e predispõe os pacientes ao risco de desenvolver síndrome metabólica (SM).
A SM é um transtorno clínico caracterizado por alterações metabólicas e hemodinâmicas ${ }^{(8)}$. Várias definições para essa síndrome têm sido propostas, porém não existe um consenso sobre qual o melhor critério a ser adotado para seu diagnóstico. A proposta do National Cholesterol Education Program Third Adult Treatment Panel (NCEP - ATP III) foi desenvolvida para uso clínico, já que não exige a comprovação de resistência à insulina, o que facilita a sua utilização ${ }^{(8-10)}$. Essa proposta tem sido utilizada em vários estudos ${ }^{(11-12)}$.

Os critérios propostos pelo NCEP - ATP III para o diagnóstico da SM são a presença de três ou mais das seguintes alterações: obesidade abdominal (circunferência abdominal - homens $\geq 102$ $\mathrm{cm}$; mulheres $\geq 88 \mathrm{~cm}$ ); triglicerídeos $\geq 150 \mathrm{mg}$ / $\mathrm{dl}$; HDL colesterol (homens $<40 \mathrm{mg} / \mathrm{dl}$; mulheres $<50 \mathrm{mg} / \mathrm{dl}$ ); pressão arterial $\geq 130 / 85 \mathrm{mmHg}$; glicemia de jejum $\geq 100 \mathrm{mg} / \mathrm{dl}^{(8-9)}$. A proposta para pacientes em DP é de avaliar o Índice de Massa Corporal (IMC) em vez de utilizar a circunferência abdominal para mensurar a obesidade, pois a presença do cateter e líquido na cavidade podem alterar a circunferência abdominal, tornando essa medida um parâmetro não confiável ${ }^{(10)}$.

O objetivo principal deste estudo foi avaliar a variação de peso de pacientes em DP durante o primeiro ano de tratamento. O objetivo secundário foi identificar pacientes com critérios para SM.

\section{MÉTODOS}

Estudo de caráter observacional, retrospectivo e quantitativo. A amostra foi composta por 35 pacientes em DP, incluindo pacientes de ambas as modalidades, ou seja, Diálise Peritoneal Ambulatorial Contínua (DPAC) ou Diálise Peritoneal Automatizada (DPA), de um hospital universitário de Porto Alegre. A coleta foi realizada no segundo semestre de 2010, por meio de prontuários dos pacientes. O critério de inclusão foi estar em programa de DP há pelo menos um ano até janeiro de 2010. Desses pacientes foram coletados os dados dos primeiros doze meses de diálise. Da amostra total, doze pacientes saíram de programa de janeiro a setembro de 2010. Dos que permaneceram, foram coletados dados atuais, ou seja, da última consulta, para identificação de critérios para SM. O instrumento de coleta de dados incluiu dados de identificação e caracterização clínico-demográfica da amostra. 
As variáveis estudadas foram: idade, sexo, doença de base, altura, início da diálise, tempo em diálise, tipo de diálise, PET, peso corporal e o IMC inicial e aos doze meses. A variável peso corporal foi coletada por trimestres, durante o primeiro ano em DP, e foi considerado o peso corporal do paciente sem o líquido de diálise peritoneal. O índice de massa corporal foi calculado baseado no peso corporal e altura, registrados em prontuário, usando-se a fórmula peso corporal em quilos, dividido pela altura em metros ao quadrado. O IMC foi classificado como menor de 20 (abaixo do peso), 20-25 (peso normal), 25,1-29,9 (sobrepeso), 30,0-39,9 (obeso), 40 ou mais (obeso mórbido) ${ }^{(13)}$.

Para identificar os pacientes com critérios para SM foram coletadas variáveis conforme os critérios do NCEP-ATP III, que são: circunferência abdominal, triglicerídeos, HDL colesterol, pressão arterial e glicemia de jejum. Os pacientes em DP podem apresentar a circunferência abdominal alterada em razão do tratamento, por isso que nesse estudo foi considerado o IMC em vez da circunferência abdominal $^{(10)}$. Tais dados são referentes ao mês de setembro de 2010, ou seja, dados atuais do paciente, independente do tempo de tratamento.

Os dados coletados foram tabulados e analisados por meio da estatística descritiva na qual as variáveis contínuas foram apresentadas como média e desvio padrão ou mediana e intervalo interquartil, conforme distribuição normal ou não. As variáveis categóricas foram apresentadas como freqüência e porcentagem.

O estudo foi aprovado pelo Comitê de Ética e Pesquisa da instituição sob o parecer 09/04760.

\section{RESULTADOS}

Foram analisados dados de 35 pacientes que estavam em diálise peritoneal no mês de janeiro de 2010. A tabela 1 apresenta as características clínicas e demográficas dos pacientes do estudo.

Nos pacientes estudados, a principal etiologia da doença renal crônica encontrada foi hipertensão arterial sistêmica (HAS) com $37 \%^{(13)}$, seguida de Diabetes mellitus (DM) com $17 \%^{(6)}$ e rins policísticos com $11 \%{ }^{(4)}$, sendo que as outras causas somaram $34 \%^{(12)}$.

A figura 1 apresenta dados do IMC inicial e do IMC após doze meses em DP. Verificou-se que $26 \%{ }^{(9)}$ dos pacientes mudaram de classificação quan-
Tabela 1 - Características clínico-demográficas dos pacientes em diálise peritoneal no primeiro ano de tratamento da Unidade de Diálise de um hospital universitário de Porto Alegre (n=35). Porto Alegre, RS, jan/set 2010.

\begin{tabular}{ll}
\hline Variável & Sumário* \\
\hline Idade (anos) & $50,8 \pm 19,5$ \\
Sexo feminino n (\%) & $22(63 \%)$ \\
DPAC n (\%) & $24(69 \%)$ \\
DPA n (\%) & $4(11 \%)$ \\
DPAC e DPA n (\%) & $7(20 \%)$ \\
Tempo em diálise n (\%) & \\
$<2$ anos & $21(60 \%)$ \\
2 a 5 anos & $12(34 \%)$ \\
$>5$ anos & $2(6 \%)$ \\
\hline
\end{tabular}

Fonte: Serviço de Arquivo Médico. Porto Alegre, RS, jan/set 2010. * Dados apresentados como: média e desvio padrão para idade; e frequência seguida de porcentagem para os demais dados.

Legenda: DPAC: Diálise Peritoneal Ambulatorial Contínua; DPA: Diálise Peritoneal Automatizada.

to ao IMC. Desses, $44 \%{ }^{(4)}$ que estavam abaixo do peso corporal passaram a ter peso corporal normal; $22 \%{ }^{(2)} \mathrm{com}$ IMC normal passaram a ter sobrepeso; $22 \%^{(2)}$ com sobrepeso passaram para obesidade e $11 \%^{(1)}$ com sobrepeso passou para o IMC normal, sendo que os demais pacientes mantiveram sua classificação inicial.

Dos 35 pacientes estudados, $71 \%{ }^{(25)}$ haviam realizado o teste do equilíbrio peritoneal. A distribuição dos pacientes conforme as categorias de transporte avaliadas pelo PET foi $12 \%\left({ }^{(3)}\right.$ alto transportador, $40 \%{ }^{(10)}$ médio alto, $44 \%^{(11)}$ médio baixo e $4 \%^{(1)}$ baixo transportador.

Na Tabela 2 consta a análise da variação de peso corporal por trimestres durante o primeiro ano de diálise peritoneal.

A média de variação de peso corporal do início do tratamento aos três meses foi de 2,2 2,3 Kg; dos

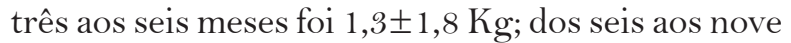
meses foi $1,1 \pm 1,5 \mathrm{Kg}$; dos nove aos doze meses foi

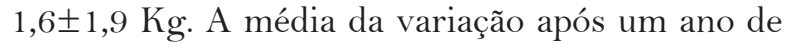
diálise foi de $3,7 \pm 3,6 \mathrm{Kg}$.

Dos 35 pacientes estudados, $34 \%{ }^{(12)}$ foram desligados do programa de diálise peritoneal a partir de janeiro de 2010 , sendo $42 \%{ }^{(5)}$ por transplante, $16 \%^{(2)}$ por peritonites e $42 \%^{(5)}$ por óbito não relacionado à diálise. 


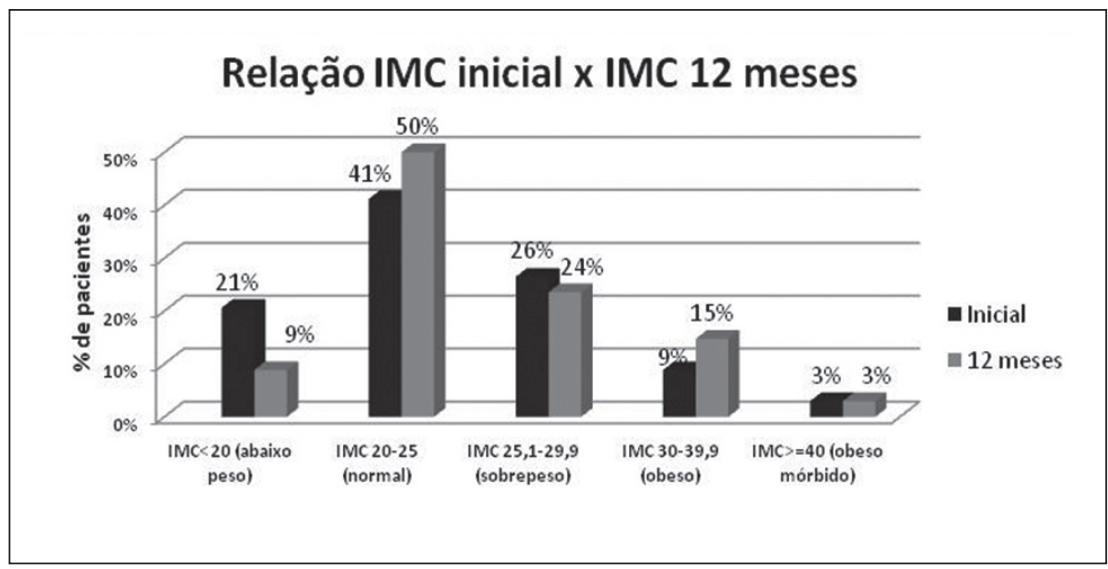

Figura 1 - Relação entre IMC inicial e IMC após 12 meses dos pacientes em diálise peritoneal de um hospital universitário de Porto Alegre (n=35). Fonte: Serviço de Arquivo Médico. Porto Alegre, RS, jan/set 2010.

Tabela $\mathbf{2}$ - Variação de peso corporal por trimestres dos pacientes em diálise peritoneal no primeiro ano de tratamento da Unidade de Diálise de um hospital universitário de Porto Alegre $(n=35)$. Porto Alegre, RS, jan/set 2010.

\begin{tabular}{ll}
\hline Variação de Peso $(\Delta \mathrm{P})$ & Sumário* $^{*}$ \\
\hline$\Delta \mathrm{P}$ inicial-3 meses & $1,8(\mathrm{O}-7,7)$ \\
$\Delta \mathrm{P}$ 3-6 meses & $0,7(\mathrm{O}-8,4)$ \\
$\Delta \mathrm{P}$ 6-9 meses & $0,5(0-5,5)$ \\
$\Delta \mathrm{P}$ 9-12 meses & $0,8(\mathrm{O}-13,5)$ \\
$\Delta \mathrm{P}$ inicial-12 meses & $2,5(\mathrm{O}-12,1)$ \\
\hline
\end{tabular}

Fonte: Serviço de Arquivo Médico. Porto Alegre, RS, jan/set 2010. * Dados apresentados como mediana e intervalo interquartil.

Dos 23 pacientes que permaneceram no programa de diálise peritoneal foram coletados dados para a identificação do risco de síndrome metabólica. Conforme conceito do NCEP-ATP III, 30\% ${ }^{(7)}$ dos pacientes tinham critérios para SM, sendo que nestes as variáveis IMC e triglicerídeos foram encontradas acima do limite de normalidade. A terceira variável mais frequente foi a HAS.

Os dados referentes à relação da variação de peso corporal inicial e atual com o tempo de diálise são apresentados na Tabela 3. A média da variação de peso corporal dos pacientes com até um ano de diálise foi $3,7 \pm 3,6 \mathrm{Kg}$, com menos de dois anos foi

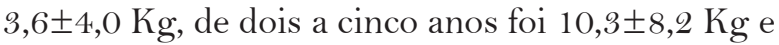
com mais de cinco anos foi $10,5 \pm 0 \mathrm{Kg}$.
Tabela 3 - Relação entre o tempo de diálise e a variação de peso corporal dos pacientes em diálise peritoneal da Unidade de Diálise de um hospital universitário de Porto Alegre (n=23). Porto Alegre, $\mathrm{RS}$, jan/set 2010.

\begin{tabular}{ll}
\hline Tempo de Diálise & Variação de Peso* \\
\hline 1 ano & $2,5(0-12,1)$ \\
$<2$ anos & $2,6(0-12,6)$ \\
2 a 5 anos & $8,7(0-20,8)$ \\
$>5$ anos & $10,5(10,5-10,5)$ \\
\hline
\end{tabular}

Fonte: Serviço de Arquivo Médico. Porto Alegre, RS, jan/set 2010. * Dados apresentados como mediana e intervalo interquartil.

\section{DISCUSSÃO}

A média de idade encontrada no presente estudo foi de 50,8 $\pm 19,5$ anos, semelhante a outros estudos brasileiros ${ }^{(14-15)}$. Encontrou-se uma prevalência de pacientes do sexo feminino, o que também foi verificado em outra pesquisa ${ }^{(15)}$, mas que diferiu de outro estudo brasileiro ${ }^{(14)}$.

Em relação ao tipo de diálise, a maioria dos pacientes, $69 \%$, realizou durante o primeiro ano a modalidade de DPAC, sendo que $20 \%$ da população estudada modificou o tipo de diálise durante o primeiro ano, passando de DPAC para DPA ou vice-versa. Esta mudança de modalidade durante o primeiro ano é decorrente de ajustes que se fazem necessários para a adequação da terapia após a realização do PET. 
O uso de DPA é indicado para pacientes com tipo de transporte peritoneal alto ou alto médio, ou para algumas situações sociais que possam beneficiar o paciente e/ou família. No entanto, um estudo de revisão da diálise peritoneal na América Latina mostrou que quase $40 \%$ dos pacientes realizavam $\mathrm{DPA}^{(16)}$. Em um estudo multicêntrico brasileiro de diálise peritoneal, a prevalência de DPA foi de $56 \%{ }^{(15)}$.

Pode-se observar que a principal doença de base encontrada foi HAS com 37\% seguido por DM com $17 \%$, sendo que as demais patologias somaram $45 \%$. No entanto, a principal etiologia da doença renal, em outro estudo brasileiro, foi nefropatia diabética seguida de nefroesclerose hipertensiva ${ }^{(15)}$.

A distribuição dos pacientes conforme o IMC foi avaliada no início do tratamento e após doze meses. Percebeu-se que houve uma redução dos pacientes com baixo peso $(\mathrm{IMC}<20)$ e sobrepeso (IMC de 25,1 a 29,9) e um aumento dos pacientes com IMC normal (IMC de 20 a 25) e obesos (IMC de 30 a 39,9). Evidenciou-se que os pacientes ganharam peso durante o primeiro ano, pois passaram de baixo peso para peso normal e os que estavam com sobrepeso passaram a ser obesos. Após doze meses, em torno de $40 \%$ dos pacientes apresentava sobrepeso e/ou obesidade. Este fato pode ser explicado pela própria manifestação da doença renal crônica quando a uremia leva a manifestações de inapetência e alteração no paladar, interferindo na ingestão de alimentos. O início da diálise reduz ou elimina estas manifestações permitindo ao doente um retorno à alimentação habitual e recuperação do apetite e peso corporal. Pode-se observar, então, que houve um aumento de pacientes com o peso normal e com obesidade e uma diminuição dos pacientes abaixo do peso e com sobrepeso.

No presente estudo a maioria dos pacientes em diálise peritoneal eram eutróficos, achado divergente de relato anterior que mostrou predomínio de sobrepeso nestes pacientes ${ }^{(14)}$. A distribuição percentual dos indivíduos que apresentavam sobrepeso ou obesidade na amostra estudada foi semelhante ao estudo observacional realizado no Brasil com pacientes em diálise peritoneal (BRAZPD) ${ }^{(15)}$. Conforme dados do Ministério da Saúde, em 2009, 46,6\% da população adulta brasileira tinha excesso de peso corporal e 13,9\% era obesa ${ }^{(1)}$. Comparando-se os dados do Ministério da Saúde com os achados do atual estudo, pode-se observar que a porcentagem de pacientes com sobrepeso e/ou obesidade é menor do que na população brasileira. Portanto, o ganho de peso corporal apresentado pelos pacientes em diálise peritoneal pode não estar associado à glicose proveniente do dialisato, mas ser uma tendência natural do indivíduo conforme o avanço da idade.

Existe discordância com relação ao peso corporal e à sobrevida dos pacientes em diálise peritoneal. Há relatos mostrando que a obesidade foi um fator significativo para aumentar a sobrevida dos pacientes em DP, sendo também associada com a redução do risco de morte desses pacientes ${ }^{(17)}$. Contudo, outros autores sugerem que estes resultados são conflitantes e esclarecem que um IMC elevado pode ser deletério para o paciente, sendo que o ideal é a pessoa estar com o peso normal para a estatura $^{(18)}$.

A distribuição das categorias do teste de equilíbrio peritoneal em nosso estudo foi semelhante a de um estudo coreano com 40,2\% médio-baixo e 36,7\% médio-alto ${ }^{(7)}$. Já em estudo chinês foi encontrado 22\% médio-baixo e 40\% médio-alto ${ }^{(19)}$. A distribuição do PET com um número pequeno de pacientes alto transportadores justifica a proporção de DPAC/DPA neste estudo, já que pacientes classificados pelo PET como médio transportadores podem ser tratados com segurança com DPAC. Dados confirmam que os pacientes alto transportadores absorvem a glicose mais rápido ${ }^{(6)}$. Em vista disso, acredita-se que esses pacientes podem apresentar maior ganho de peso, no entanto, em nosso estudo, devido ao pequeno número de pacientes alto transportadores, não foi possível realizar essa análise.

Nosso estudo mostrou que a variação do peso corporal após o primeiro ano e em especial no primeiro trimestre corresponde a uma mudança de $3,5 \%$ do peso corporal. Resultados oriundos de um estudo sueco, que avaliou as mudanças no peso corporal dos pacientes em DPAC, encontraram uma mudança de 1,9\% no peso corporal médio, quando observado em média sete meses após o início do tratamento $^{(2)}$. O valor encontrado nesse estudo foi inferior ao da presente pesquisa, mas o tempo de observação também foi menor. Quando analisados os dois primeiros anos, verifica-se que o maior ganho de peso foi no primeiro ano de diálise, pois a média em dois anos é praticamente a mesma encontrada no primeiro ano. Muitas interpretações podem advir deste ganho de peso, uma destas pode ser simplesmente que o tratamento com a diálise minimiza os sintomas da uremia restaurando o apetite e favore- 
cendo o ganho de peso. Talvez, se comparássemos o ganho de peso não com o início da diálise mas com o valor antes do aparecimento da doença renal este ganho seria nulo ou até mesmo inexistente.

Infelizmente as taxas de saída do tratamento ainda são muito altas no Brasil, estas podem mudar conforme a experiência do centro de diálise, quanto mais experiente a unidade de diálise mais confiante é a equipe para o manejo das complicações mais comuns. $\mathrm{O}$ fato da unidade em estudo ter a DP como forma de tratamento para $35 \%$ dos seus doentes pode estar relacionada com a alta percentagem de saída por transplante, considerada uma saída positiva. $O$ percentual de pacientes desligados foi semelhante aos dados do BRAZPD ${ }^{(15)}$, porém, o principal motivo de saída foi o óbito por causas cardiovasculares. Em outro estudo que acompanhou os pacientes em diálise peritoneal por 10 anos, o principal motivo de saída também foi o transplante renal ${ }^{(20)}$.

De acordo com a primeira diretriz brasileira de diagnóstico e tratamento da SM, os pacientes que apresentam critérios para SM têm maior chance de mortalidade cardiovascular do que a população em geral $^{(9)}$. Em um estudo realizado em São Paulo, que avaliou 200 pacientes em acompanhamento cardiológico, a taxa de SM encontrada, pelos critérios da NCEP-ATP III, foi de $35,5 \%$, com destaque para alteração de glicemia, triglicerídeos e $\operatorname{HAS}^{(11)}$; em outro estudo realizado no RS, também com pacientes cardiológicos, encontrou-se uma prevalência de $61,5 \%$. Em estudos de revisão bibliográfica foi verificado que a prevalência de $\mathrm{SM}$ nos pacientes em DP é em torno de igual ou maior que $50 \%{ }^{(8,10)}$.

No presente estudo, $30 \%$ dos pacientes apresentavam critérios para $\mathrm{SM}$, sendo que as taxas de triglicerídeos alteradas e IMC fora da normalidade foram critérios presentes em todos os pacientes e o terceiro critério mais encontrado foi HAS. Nota-se que a nossa amostra apresentava uma prevalência menor de SM em relação aos outros estudos sobre essa temática. Entretanto, esse dado é importante, já que os pacientes que apresentam SM têm elevada morbidade.

Em relação ao tempo de tratamento, $60 \%$ estão em diálise peritoneal há menos de dois anos. Apenas 6\% está há mais de cinco anos. O baixo número de pacientes há mais tempo na terapia de substituição renal deve-se à alta mortalidade da doença renal crônica. A média da variação de peso corporal atual e do peso corporal inicial relacionada ao tempo de diálise apontou que a maior variação de peso corporal ocorreu entre dois e cinco anos do início da DP.

\section{CONCLUSÕES}

Os dados sugerem que o ganho de peso corporal mais importante ocorre durante o primeiro ano de diálise peritoneal, pois no decorrer do tratamento o aumento de peso corporal segue a tendência natural da população brasileira. Há uma alta incidência de SM nos pacientes em diálise peritoneal, o que pode aumentar a morbimortalidade destes pacientes.

O acompanhamento e avaliação do enfermeiro são de fundamental importância para o sucesso da terapia de diálise peritoneal. A educação continuada favorece a adesão, estimula o auto cuidado e melhora da qualidade de vida desses pacientes. Faz parte das atribuições do enfermeiro avaliar e identificar precocemente os fatores de risco para o desenvolvimento de SM para minimizar as comorbidades e melhorar a qualidade de vida do indivíduo.

\section{REFERÊNCIAS}

1 Ministério da Saúde (BR). Notícias. Quase metade dos brasileiros tem excesso de peso [Internet]. Brasília; 2010 [citado 2010 novembro 10]. Disponível em: http://portal.saude.gov.br/portal/aplicacoes/ noticias/default.cfm? pg=dspDetalheNoticia\&id area $=124 \&$ CO_NOTICIA $=11458$.

2 Fernström A, Hylander B, Mortiz A, Jacobsson H, Rössner S. Increase of intra-abdominal fat in patients treated with continuous ambulatory peritoneal dialysis. Perit Dial Int. 1998;18: 166-71.

3 Levy MN, Koeppen BM, Station BA. Berne \& Levy, Fundamentos de Fisiologia. Rio de Janeiro: Elsevier; 2006. p. 617-25.

4 Machado UF, Schaan BD, Seraphim PM. Transportadores de glicose na síndrome metabólica. Arq Bras Endocrinol Metab. 2006;50(2): 177-89.

5 Fortes PC, Moraes TP, Mendes JG, Stinghen AE, Ribeiro SC, Pecoits-Filho R. Insulin resistance and glucose homeostasis in peritoneal dialysis. Perit Dial Int. 2009; 29 (Suppl 2): 145-48.

6 Junior MAG, Andreoli MCC, Sardenberg CS, Santos BFC, Neto MC. Diálise no paciente com insuficiência renal crônica: hemodiálise e diálise peritoneal. 
In: Barros E. Nefrologia rotinas, diagnósticos e tratamento. Porto Alegre: Artmed; 2006. p. 424-41.

7 Kang D, Yoon K, Choi K, Lee R, Lee H, Han D, et al. Relationship of peritoneal membrane transport characteristics to the nutritional status in CAPD patients. Nephrol Dial Transplant. 1999;14: 1715-22.

8 Park SH, Lindholm B. Definition of metabolic syndrome in peritoneal dialysis. Perit Dial Int. 2009; 29 (Suppl 2): 137-44.

9 Sociedade Brasileira de Cardiologia. I diretriz brasileira de diagnóstico e tratamento da síndrome metabólica. Arq Bras Cardiol. 2005;84 (Suppl 1): 02-28.

10 Li PKT, Kwan BCH, Ko GTC, Chow KM, Leung $\mathrm{CB}$, Szeto CC. Treatment of metabolic syndrome in peritoneal dialysis patients. Perit Dial Int. 2009; 29 (Suppl 2): 149-52.

11 Nakazone MA, Pinheiro A, Braile MCVB, Pinhel MAS, Sousa GF, Junior SP, et al. Prevalência de síndrome metabólica em indivíduos brasileiros pelos critérios de NCEP-ATPIII e IDF. R Assoc Med Bras. 2007;53(5): 407-13.

12 Bopp M, Barbiero S. Prevalência de síndrome metabólica em pacientes de um ambulatório do Instituto de Cardiologia do Rio Grande do Sul (RS). Arq Bras Cardiol. 2009;93(5): 473-77.

13 WHO (World Health Organization). Physical Status: the use and interpretation anthropometry. WHO Tech Rep Series. 1995; 854:1-36.
14 Vasselai P, Kamimura MA, Bazanelli AP, Pupim LB, Avesani CM, Ribeiro FSM, et al. Factors associated with body-fat changes in prevalent peritoneal dialysis patients. J Ren Nutr. 2008:18(4): 363-69.

15 Fernandes N, Bastos MG, Filho RP, Pereira LC, Franco M, Haddad AS, et al. Sucessos e fracassos - uma análise dos dados do BRAZPD comparados às diretrizes espanholas. J Bras Nefro. 2008;30(1): 22-31.

16 Pecoits-Filho R, Abensur H, Cueto-Manzano AM, Dominguez J, Filho JCD, Fernandez-Cean J, et al. Overview of peritoneal dialysis in latin America. Perit Dial Int. 2007;27(3): 316-21.

17 Johnson DW, Herzig KA, Purdie DM, Chang W, Brown AM, Rigby RJ, et al. Is obesity a favorable prognostic factor in peritoneal dialysis patients? Perit Dial Int. 2000;20(6): 715-21.

18 Johnson DW. What is the optimal fat mass in peritoneal dialysis patients? Perit Dial Int. 2007;27 (Suppl 2): $250-54$.

19 Yu X, Zhou Y, Feng L, Zhang D, Liu W. Influence of peritoneal transfer status on fasting blood glucose in non-diabetic nephropathy patients on continuous ambulatory peritoneal dialysis. Chin Med J 2009; 122(24): 2977-80.

20 Kolesnyk I, Dekker FW, Boeschoten EW, Krediet RT. Time-dependent reasons for peritoneal dialysis technique failure and mortality. Perit Dial Int. 2010;30(2): $170-77$.

\author{
Endereço do autor / Dirección del autor / \\ Author's address: \\ Ana Elizabeth Prado Lima Figueiredo \\ Rua Miracema, 407, Chácara das Pedras \\ 91330-490, Porto Alegre, RS \\ E-mail: anaef@pucrs.br
}

